\title{
Breakout Group A: What should the research questions and priorities in paleoscience be for the next 10 years?
}

Sylvia Dee ${ }^{1}$ and Francesco Muschitiello ${ }^{2}$

'Department of Geological Sciences, University of Southern California, Los Angeles, USA; sdee@usc.edu

${ }^{2}$ Department of Geological Sciences, Stockholm University, Sweden

$\mathrm{H}$ ow can we improve model-based estimates and predictions? How can we improve the production of paleo data? How can we better constrain past rates of change in the Earth system? These questions, among others, were identified as key priorities for future paleoscience during our breakout sessions at the YSM.

We identified that model-based climate sensitivity estimates and the ability to correctly capture climate feedbacks, abrupt transitions, and threshold behavior in models are key to predicting climate and associated changes. Integrated earthsystem modeling with improved feedback interactions will be required to study whole-earth system dynamics.

Furthermore, assessing climate model performance requires better datasets of high-resolution proxy reconstructions: We need more high-quality data from under-represented regions. We also need new proxies for several climate variables. Also high on our wish list are solid constraints upon previously unresolved climate system components such as clouds and aerosols. We require better solar and volcanic forcing reconstructions, and we should strive to understand the underlying causes of discrepancies between the different forcing reconstructions available.

To improve the quality of our proxy networks we need to employ replication, high-resolution dating, statistical analysis and multi-proxy approaches in our research. Data uncertainty estimates should always be clearly stated. Process studies and controlled experiments must be used to establish regional calibrations and transfer functions to allow proxy-based reconstructions to capture not only highfrequency climate variability, but also a quantifiable climatic parameter such as temperature or precipitation.

Finally, we need to compile datasets and make them available in a quality-controlled, well-documented and easy-to-use form. Strict formats for "big data" should be employed in a globally acknowledged framework. The field could vastly benefit from larger collaboration with computer software engineers and informatics science to improve efficiency and manageability of earth science datasets.

\section{Breakout Group B: Advocating the relevance of paleo- research to a funding agency or policy maker}

Ilham Bouimetarhan ${ }^{1}$ and Hans Christian Steen-Larsen ${ }^{2}$

'MARUM-Center for Marine Environmental Sciences, University of Bremen, Germany; bouimetarhan@uni-bremen.de ${ }^{2}$ Cooperative Institute for Research in Environmental Sciences, University of Colorado, Boulder, USA

ear policy makers and funders of sci-
ence,

We understand your need to base your decisions and investments on stronger arguments of the important role that paleo-research plays in international efforts to understand and emphasize the social, economic, and geopolitical implications of Earth's changing climate. We are also acutely aware of many unanswered questions and substantial uncertainties that currently exist, and always will exist, in paleo-research, as in any other field of science. However, it is now evident that better projections of future climate and environmental change, which form the basis of decisions on national and international greenhouse gas emission policies, require consultation with information from Earth's past.

Repeatedly during its history, Earth's climate has changed abruptly within just a few decades. Climatic variability including changes in the magnitude and frequency of extreme events such as droughts and floods has often had a devastating impact on local societies, and events in the future are likely to bring greater environmental risks as the environment is already subject to substantial stress. Furthermore, due to the increased complexity of modern societies extreme events are likely to become more costly, as recently illustrated by Hurricane Sandy's effect on New York City.

Paleoscientists are undertaking enormous efforts to assess the high complexity of Earth's climate system and gain a better understanding of the general forces controlling global climate change. Integrating local and regional climate information from marine and terrestrial environments all over the world has allowed, for example, the identification of important feedbacks in the climate system; this is crucial if we are to avoid being surprised by abrupt climatic events. The results of this research have helped us to better understand the role human activities play in causing a large part of the changes in the Earth's climate system, namely the significant increase in global temperatures. Moreover, many paleoclimate results are now being effectively assimilated with climate models in order to provide better future projections and predictions of potential impacts likely to affect people in the short-term and in coming decades.

While paleoscientific research is able to highlight some of the environmental risks threatening our planet, much remains to be learned, and this kind of research will still need more financial investment in order to thoroughly examine other scientific questions. While it might not provide direct applications, or solutions for engineering a better future, paleoscientific research makes a substantial contribution to constraining the possible scenarios of future environmental and climatic changes. We encourage you therefore to let paleoscience evidence guide you towards wise decisions on policy and science funding in the context of the high priority challenges facing humanity.

Yours sincerely,

Ilham Bouimetarhan and Hans Christian Steen-Larsen

On behalf of the PAGES Young Scientists 2013 